\title{
On the Evaluation of Riemann Zeta Functions for Even Integers
}

\author{
Ribhu Paul \\ School of Physical Sciences, IACS, Kolkata, India
}

\begin{abstract}
A recursive method for obtaining the Zeta function for even integers is obtained starting from the Fourier series expansion of the function $f(x)=x$. Repeating the method after term by term integration yields the final, simplified closed form that happens to be a recursion relation. Using the obtained recursion relation, one can successively evaluate the values of zeta functions for even integers.
\end{abstract}

\section{Introduction}

The zeta function, usually referred to as the Riemann zeta function has been studied in many different forms for centuries. The harmonic series, $\zeta(1)$ was proven to be divergent as far back as the 14th century. In the 18th century, the Swiss mathematician Leonhard Euler found a closed form expression for the sum of the reciprocals of the squared integers i.e. $\zeta(2)$. He also generalized this result and found a closed form expression for $\zeta(2 n)$ for $n \in N$. In the 19th century, the German mathematician Bernhard Riemann considered $\zeta$ as a complex function. He published his work in the 1859 paper "On the Number of Primes Less Than a Given Magnitude", which is one of the most influential works of modern mathematics. When using mathematical symbols to describe the Riemann zeta function, it is represented as an infinite series:

$$
\zeta(s)=\sum_{n=1}^{\infty} n^{-s}, \operatorname{Re}(s)>1
$$


where $R e(s)$ is the real part of the complex number $s$. For example, if $s=a+i b$, then $\operatorname{Re}(s)=a$ (where $i^{2}=-1$ ). Hence, the series when expanded out would be:

$$
\frac{1}{1^{s}}+\frac{1}{2^{s}}+\frac{1}{3^{s}}+\ldots \text { ad infinitum }
$$

The purpose of this article is to systematically introduce a recursive method for evaluating zeta functions for even integers which has been investigated from the perspective of Fourier series.

\section{Method}

Fourier Series Integration Lemma:- Fourier series of a piece-wise smooth function $f(x)$ can always be integrated term by term and the result is a convergent infinite series that always converges to the integral of $f$ in $x \in[-L, L]$, where the period is $2 L$ [2].

Remark:- The lemma states that a Fourier series can be integrated term by term and that the result would be a convergent infinite series which would converge to the integral of $f$.However, it may not be a formal Fourier series and the obtained series can be re-arranged as a Fourier series expansion of the integral of the function $f$.

The following parts solely focus on the evaluation of Zeta functions for even integers by rigorous method of Fourier term by term integration which simplifies into a closed form.

One could consider $f(x)=x$ in $[-\pi, \pi]$. Now, one can express the function in the range $(-\infty, \infty)$ by maintaining a period $\pi$ which can be obtained by doing a Fourier series expansion of the function $f(x)$ stated above as:-

$$
f(x)=a_{0}+\sum_{n=1}^{\infty} a_{n} \cos (n x)+\sum_{n=1}^{\infty} b_{n} \sin (n x)
$$

where

$$
\begin{gathered}
a_{0}=\frac{1}{2 \pi} \int_{-\pi}^{-\pi} x d x=0 \\
a_{n}=\frac{1}{2 \pi} \int_{-\pi}^{-\pi} x \cos (n x) d x=\frac{1}{2 \pi}\left[\frac{x \sin (n x)}{n}\right]_{-\pi}^{-\pi}-\frac{1}{n \pi} \int_{-\pi}^{-\pi} \sin (n x) d x=0
\end{gathered}
$$


R. Paul

and

$$
\begin{aligned}
b_{n} & =\frac{1}{2 \pi} \int_{-\pi}^{-\pi} x \sin (n x) d x=\frac{1}{2 \pi}\left[-\frac{x \cos (n x)}{n}\right]_{-\pi}^{-\pi}+\frac{1}{n \pi} \int_{-\pi}^{-\pi} \cos (n x) d x \\
& =\frac{1}{n \pi} 2 \pi(-1)^{n+1}+0=\frac{2}{n}(-1)^{n+1}
\end{aligned}
$$

Hence,

$$
f(x)=x=\sum_{n=1}^{\infty} \frac{2}{n}(-1)^{n+1} \sin (n x)
$$

in $[-\pi, \pi]$.Now one can use term by term integration of (1) to obtain another valid series :-

$$
\frac{x^{2}}{2}=C_{0}+\int \sum_{n=1}^{\infty} \frac{2}{n}(-1)^{n+1} \sin (n x)
$$

where $C_{0}$ is a constant of integration.

So,

$$
\begin{aligned}
& \frac{x^{2}}{2}=C_{0}+\sum_{n=1}^{\infty} \frac{2}{n}(-1)^{n+1} \int \sin (n x) d x \\
& \text { or, } \\
& x^{2}=2 C_{0}+\sum_{n=1}^{\infty} \frac{4}{n^{2}}(-1)^{n+2} \cos (n x)
\end{aligned}
$$

Now in order to obtain $2 C_{0}$, one can integrate this from $-\pi$ to $\pi$ to obtain,

$$
\frac{1}{2 \pi} \int_{-\pi}^{-\pi} x^{2} d x=2 C_{0}+\sum_{n=1}^{\infty} \frac{4}{n^{2}}(-1)^{n+2} \int_{-\pi}^{-\pi} \cos (n x) d x
$$

Thus, one finds,

$$
2 C_{0}=\frac{\pi^{2}}{3}
$$

Thus, from the previous results, one concludes:-

$$
x^{2}=\frac{\pi^{2}}{3}+\sum_{n=1}^{\infty} \frac{4}{n^{2}}(-1)^{n+2} \cos (n x)
$$


in $[-\pi, \pi]$. Now putting $x=\pi$ one obtains,

$$
\pi^{2}=\frac{\pi^{2}}{3}+\sum_{n=1}^{\infty} \frac{4}{n^{2}}(-1)^{n}(-1)^{n}
$$

or,

$$
\sum_{n=1}^{\infty} \frac{1}{n^{2}}=\frac{\pi^{2}}{6}
$$

or,

$$
\zeta(2)=\frac{\pi^{2}}{6}
$$

Now, applying term by term integration and substituting the Fourier series for $x$ in the final form, one obtains the Fourier-like series of the function $x^{3}$ in the same range as follows:-

$$
x^{3}=2 \pi^{2} \sum_{n=1}^{\infty} \frac{(-1)^{n+1}}{n} \sin (n x)+12 \sum_{n=1}^{\infty} \frac{(-1)^{n+2}}{n^{4}} \sin (n x)
$$

Hence, it is observed that if we have $x^{n}$ as the function in the interval considered here $([-\pi, \pi])$, then for $n$ being odd, $a_{0}$ term of the Fourier series drops out and when $n$ is even, i.e. $n=2 m$ [where $\left.m \in \mathbb{I}^{+}\right], a_{0}=2 C_{0}=\frac{\pi^{2 m}}{2 m+1}$ . So, from equation (4) $x^{4}$ in the interval considered is obtained as:-

$$
x^{4}=\frac{\pi^{4}}{5}+(3 \times 8) \sum_{n=1}^{\infty} \frac{(-1)^{n+2}}{n^{2}} \cos (n x)+48 \sum_{n=1}^{\infty} \frac{(-1)^{n+3}}{n^{4}} \cos (n x)
$$

in $[-\pi, \pi]$. By plugging in $x=\pi$ in $(4)$ :-

$$
\pi^{4}=\frac{\pi^{4}}{5}+8 \pi^{2} \sum_{n=1}^{\infty} \frac{(-1)^{n}(-1)^{n}}{n^{2}}+48 \sum_{n=1}^{\infty} \frac{(-1)^{n}(-1)^{n}}{n^{4}}(-1)
$$

or,

$$
\frac{4 \pi^{4}}{5}=8 \pi^{2} \times \zeta(2)+(-48) \times \zeta(4)
$$

or,

$$
\zeta(4)=\frac{\pi^{4}}{90}
$$


From (2) we observe that the number of closed form expressions with $\sum_{n=1}^{\infty}$ appear once and from (4) we observe that the number of closed form expressions with $\sum_{n=1}^{\infty}$ appear twice.

So, quite naturally it is expected that,

$$
x^{6}=\frac{\pi^{6}}{7}+A_{1} \sum_{n=1}^{\infty} \frac{g_{1}(n x)}{n^{2}}+A_{2} \sum_{n=1}^{\infty} \frac{g_{2}(n x)}{n^{4}}+A_{3} \sum_{n=1}^{\infty} \frac{g_{3}(n x)}{n^{6}}
$$

where $A_{1}, A_{2}$ and $A_{3}$ are real numbers and $g_{1}, g_{2}$ and $g_{3}$ are unknown functions at this stage which can be determined from proceeding further as before. Thus, more generally it can be claimed that,

$$
\begin{aligned}
x^{2 m+1} & =\frac{\pi^{2 m}}{2 m+1}+A_{1} \sum_{n=1}^{\infty} \frac{g_{1}(n x)}{n^{2}}+A_{2} \sum_{n=1}^{\infty} \frac{g_{2}(n x)}{n^{4}} \\
& +A_{3} \sum_{n=1}^{\infty} \frac{g_{3}(n x)}{n^{6}}+\ldots A_{m} \sum_{n=1}^{\infty} \frac{g_{m}(n x)}{n^{2 m}}
\end{aligned}
$$

as a standard form $\left\{A_{k}: \mathrm{k}=1,2, \ldots \mathrm{m}\right\}$ and $\left\{g_{k}: \mathrm{k}=1,2, \ldots \mathrm{m}\right\}$ are to be found. The Fourier series of $x^{5}$ and thereafter $x^{6}$ in the concerned interval are to be evaluated in a very similar way (by successive term by term integration and substitution of previously obtained Fourier series wherever its needed). Thus, it follows that:-

$$
\begin{aligned}
x^{5} & =2 \pi^{4} \sum_{n=1}^{\infty} \frac{(-1)^{n+1}}{n} \sin (n x)+\frac{5 !}{3} \pi^{2} \sum_{n=1}^{\infty} \frac{(-1)^{n+2}}{n^{3}} \sin (n x) \\
& +5 ! \times 2 \sum_{n=1}^{\infty} \frac{(-1)^{n+3}}{n^{5}} \sin (n x)
\end{aligned}
$$

in $[-\pi, \pi]$ and

$$
\begin{aligned}
x^{6} & =\frac{\pi^{6}}{7}+6 \times 2 \pi^{4} \sum_{n=1}^{\infty} \frac{(-1)^{n+2}}{n^{2}} \cos (n x)+\frac{6 !}{3} \pi^{2} \sum_{n=1}^{\infty} \frac{(-1)^{n+3}}{n^{4}} \cos (n x) \\
& +6 ! \times 2 \sum_{n=1}^{\infty} \frac{(-1)^{n+4}}{n^{6}} \cos (n x)
\end{aligned}
$$


in $[-\pi, \pi]$.

plugging in $x=\pi$ in (7), one obtains,

$$
\zeta(6)=\frac{\pi^{6}}{945}
$$

Identically proceeding further one can obtain,

$$
\begin{aligned}
x^{7} & =2 \pi^{6} \sum_{n=1}^{\infty} \frac{(-1)^{n+1}}{n} \sin (n x)+7 \times 6 \times 2 \pi^{4} \sum_{n=1}^{\infty} \frac{(-1)^{n+2}}{n^{3}} \sin (n x) \\
& +\frac{7 !}{3} \pi^{2} \sum_{n=1}^{\infty} \frac{(-1)^{n+3}}{n^{5}} \sin (n x)+7 ! \times 2 \sum_{n=1}^{\infty} \frac{(-1)^{n+5}}{n^{7}} \sin (n x)
\end{aligned}
$$

in $[-\pi, \pi]$. And thereafter from (8),

$$
\begin{aligned}
x^{8} & =\frac{\pi^{8}}{9}+8 \times 2 \pi^{6} \sum_{n=1}^{\infty} \frac{(-1)^{n+2}}{n^{2}} \cos (n x) \\
& +8 \times 7 \times 6 \times 2 \pi^{4} \sum_{n=1}^{\infty} \frac{(-1)^{n+3}}{n^{4}} \cos (n x) \\
& +\frac{8 !}{3} \pi^{2} \sum_{n=1}^{\infty} \frac{(-1)^{n+4}}{n^{6}} \cos (n x)+8 ! \times 2 \sum_{n=1}^{\infty} \frac{(-1)^{n+5}}{n^{8}} \cos (n x)
\end{aligned}
$$

in $[-\pi, \pi]$ is obtained in a straightforward manner.

Substituting $x=\pi$ in (9), and using the values of $\zeta(2), \zeta(4)$ and $\zeta(6)$, one obtains,

$$
\zeta(8)=\frac{\pi^{8}}{9450}
$$

Now, that the zeta functions for the first four even integers are found, a predictable pattern emerges that can be exploited to a point where one doesn't require the explicit term by term integration. This can only be achieved when the explicit form of (5) is known. Before anything can be concluded, it is important to summarize what have been obtained from the beginning as follows:- 
R. Paul

$$
x^{2 \cdot 1}-\frac{\pi^{2 \cdot 1}}{2 \cdot 1+1}=2 ! \times \sum_{n=1}^{\infty} \frac{2}{n^{2}}(-1)^{n+2} \cos (n x)
$$

$$
\begin{aligned}
x^{2 \cdot 2}-\frac{\pi^{2 \cdot 2}}{2 \cdot 2+1}= & 4 \times \pi^{2} \sum_{n=1}^{\infty} \frac{2}{n^{2}}(-1)^{n+2} \cos (n x) \\
& +2 ! \times \sum_{n=1}^{\infty} \frac{2}{n^{4}}(-1)^{n+3} \cos (n x)
\end{aligned}
$$

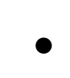

$$
\begin{aligned}
x^{2 \cdot 3}-\frac{\pi^{2 \cdot 3}}{2 \cdot 3+1} & =6 \times \pi^{4} \sum_{n=1}^{\infty} \frac{2}{n^{2}}(-1)^{n+2} \cos (n x) \\
& +(6 \times 5 \times 4) \times \pi^{2} \sum_{n=1}^{\infty} \frac{2}{n^{4}}(-1)^{n+3} \cos (n x) \\
& +6 ! \times \sum_{n=1}^{\infty} \frac{2}{n^{6}}(-1)^{n+4} \cos (n x)
\end{aligned}
$$

•

$$
\begin{aligned}
x^{2 \cdot 4}-\frac{\pi^{2 \cdot 4}}{2 \cdot 4+1} & =8 \times \pi^{6} \sum_{n=1}^{\infty} \frac{2}{n^{2}}(-1)^{n+2} \cos (n x) \\
& +(8 \times 7 \times 6) \times \pi^{4} \sum_{n=1}^{\infty} \frac{2}{n^{4}}(-1)^{n+3} \cos (n x) \\
& +(8 \times 7 \times 6 \times 5) \times \pi^{2} \sum_{n=1}^{\infty} \frac{2}{n^{6}}(-1)^{n+4} \cos (n x) \\
& +8 ! \times \sum_{n=1}^{\infty} \frac{2}{n^{8}}(-1)^{n+5} \cos (n x)
\end{aligned}
$$

where the interval of interest is $[-\pi, \pi]$ for all of the above.

Quite clearly, one can generalise the above results for any arbitrary positive integer value $m$ as:- 


$$
\begin{aligned}
x^{2 m}-\frac{\pi^{2 m}}{2 m+1} & =\frac{(2 m) !}{(2 m-1) !} \pi^{2 m-2} \sum_{n=1}^{\infty} \frac{(-1)^{n+2}}{n^{2}} \phi(n x) \\
& +\frac{(2 m) !}{(2 m-3) !} \pi^{2 m-4} \sum_{n=1}^{\infty} \frac{(-1)^{n+3}}{n^{4}} \phi(n x) \\
& +\frac{(2 m) !}{(2 m-5) !} \pi^{2 m-6} \sum_{n=1}^{\infty} \frac{(-1)^{n+4}}{n^{6}} \phi(n x)+\ldots \\
& +\frac{(2 m) !}{3 !} \pi^{2} \sum_{n=1}^{\infty} \frac{(-1)^{n+m}}{n^{2 m-2}} \phi(n x)+\frac{(2 m) !}{1 !} \pi^{0} \sum_{n=1}^{\infty} \frac{(-1)^{n+m+1}}{n^{2 m}} \phi(n x)
\end{aligned}
$$

in $[-\pi, \pi]$ where $\phi(n x)=2 \cos (n x)$. Taking the value of the generalised form at $x=\pi$, and noting that $\phi(n \pi)=2 \cos (n \pi)=2(-1)^{n}$, one obtains:-

$$
\begin{aligned}
\frac{m \pi^{2 m}}{2 m+1} & =\frac{(2 m) !}{(2 m-1) !} \pi^{2 m-2} \sum_{n=1}^{\infty} \frac{1}{n^{2}}(-1)^{2}+\frac{(2 m) !}{(2 m-3) !} \pi^{2 m-4} \sum_{n=1}^{\infty} \frac{1}{n^{4}}(-1)^{3} \\
& +\frac{(2 m) !}{(2 m-5) !} \pi^{2 m-6} \sum_{n=1}^{\infty} \frac{1}{n^{6}}(-1)^{4}+\ldots \ldots+\frac{(2 m) !}{3 !} \pi^{2} \sum_{n=1}^{\infty} \frac{1}{n^{2 m-2}}(-1)^{m} \\
& +\frac{(2 m) !}{1 !} \pi^{0} \sum_{n=1}^{\infty} \frac{1}{n^{2 m}}(-1)^{m+1}
\end{aligned}
$$

or,

$$
\begin{aligned}
\frac{m \pi^{2 m}}{2 m+1} & =\frac{(2 m) !}{(2 m-1) !} \pi^{2 m-2} \zeta(2)-\frac{(2 m) !}{(2 m-3) !} \pi^{2 m-4} \zeta(4)+\frac{(2 m) !}{(2 m-5) !} \pi^{2 m-6} \zeta(6) \\
& +\ldots \ldots+\frac{(2 m) !}{3 !} \pi^{2}(-1)^{m} \zeta(2 m-2)+\frac{(2 m) !}{1 !} \pi^{0}(-1)^{m+1} \zeta(2 m)
\end{aligned}
$$

or more precisely,

$$
\frac{m \pi^{2 m}}{2 m+1}=\sum_{j=1}^{m}(-1)^{j+1} \pi^{2 m-2 j} \frac{(2 m) !}{(2 m-2 j+1) !} \zeta(2 j)
$$

for $m=1$,

$$
\frac{\pi^{2}}{2 \times 3}=\zeta(2)=\frac{\pi^{2}}{6}
$$


and for $m=2$,

$$
\frac{2 \pi^{2}}{4 ! \times 5}=\frac{\pi^{2} \times \zeta(2)}{3 !}-\zeta(4)
$$

which gives,

$$
\zeta(4)=\left[-\left(\frac{2}{4 ! \times 5}\right)+\frac{1}{3 ! \times 6}\right] \pi^{4}=\frac{\pi^{4}}{90}
$$

Therefore, equation (10) generates all the even zeta functions. Although it seems to be a cumbersome method for obtaining the values of zeta functions for higher even integers, it is quite interesting to see how the zeta functions for even integers are linked together in the following way:-

$$
\begin{aligned}
\zeta(2) & =\frac{\pi^{2}}{2 ! \times 3} \\
\zeta(4) & =\frac{\pi^{2}}{3 !} \zeta(2)-\frac{2 \pi^{4}}{4 ! \times 5} \\
\zeta(6) & =-\frac{\pi^{4}}{5 !} \zeta(2)+\frac{\pi^{2}}{3 !} \zeta(4)+\frac{3 \pi^{6}}{6 ! \times 7} \\
\zeta(8) & =\frac{\pi^{6}}{7 !} \zeta(2)-\frac{\pi^{4}}{5 !} \zeta(4)+\frac{\pi^{2}}{3 !} \zeta(6)-\frac{4 \pi^{8}}{8 ! \times 9} \\
\zeta(10) & =-\frac{\pi^{8}}{9 !} \zeta(2)+\frac{\pi^{6}}{7 !} \zeta(4)-\frac{\pi^{5}}{4 !} \zeta(6)+\frac{\pi^{2}}{3 !} \zeta(8)-\frac{5 \pi^{10}}{10 ! \times 11}
\end{aligned}
$$

Thus, one can start from (10) and work out recursively to obtain all the higher even integer zeta functions. It is therefore possible to modify (10) into a recursion relation in the following manner:-

$$
\begin{aligned}
\frac{m \pi^{2 m}}{2 m+1} & =\sum_{j=1}^{m}(-1)^{j} \pi^{2 m-2 j} \frac{(2 m) !}{(2 m-2 j+1) !} \zeta(2 j) \\
\frac{m \pi^{2 m}}{2 m+1} & =\sum_{j=1}^{m-1}(-1)^{j+1} \pi^{2 m-2 j} \frac{(2 m) !}{(2 m-2 j+1) !} \zeta(2 j)+\frac{(-1)^{m+1}(2 m) !}{1 !} \zeta(2 m)
\end{aligned}
$$

As, $(2 m) !(2 m+1)=(2 m+1) !=\Gamma(2 m+2),[\Gamma$ is the Euler Gamma function $]$ 
Therefore,the following recursion relation is obtained after few algebraic manipulation:-

$$
\zeta(2 m)=(-1)^{m+1}\left[\frac{m \pi^{2 m}}{\Gamma(2 m+2)}+\sum_{j=1}^{m-1}(-1)^{j} \frac{\pi^{2 m-2 j}}{\Gamma(2 m-2 j+2)} \zeta(2 j)\right]
$$

where $m \in \mathbb{I}^{+}$. Relation (11) is the key recursion relation which has now been obtained. Now, one can notice that $\zeta(2 m)=\mathcal{B}_{2 m} \pi^{2 m}$ where $\mathcal{B}_{2 m}$ are the coefficients that are the primary subject of interest. Hence, one could also obtain,

$$
\mathcal{B}_{2 m}=(-1)^{m+1}\left[\frac{m}{\Gamma(2 m+2)}+\sum_{j=1}^{m-1}(-1)^{j} \frac{1}{\Gamma(2 m-2 j+2)} \mathcal{B}_{2 j}\right]
$$

$m \in \mathbb{I}^{+}$and $m>1$.

Now, from Euler's discovery of closed form for $\zeta(2 m)\left[m \in \mathbb{I}^{+}\right]$, it is well known that,

$$
\zeta(2 m)=(-1)^{m+1} \frac{B_{2 m}(2 \pi)^{2 m}}{2(2 m) !}
$$

from [1] where $B_{2 m}$ represents Bernoulli numbers. Hence, a one to one relation is readily obtained that relates $\mathcal{B}_{2 m}$ with $B_{2 m}$ i.e.

$$
\mathcal{B}_{2 m}=(-1)^{m+1} \frac{B_{2 m}(2)^{2 m-1}}{(2 m) !}
$$

\section{Results}

First 5 values for $\mathcal{B}_{2 m}\left[m \in \mathbb{I}^{+}\right]$are calculated below from the recursive scheme (12):-

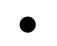

$$
\begin{gathered}
\mathcal{B}_{2}=(-1)^{2} \frac{1}{\Gamma(2 \cdot 1+2)}=\frac{1}{6} \\
\mathcal{B}_{4}=(-1)^{3}\left[\frac{2}{\Gamma(2 \cdot 2+2)}+\frac{(-1) \cdot \mathcal{B}_{2}}{\Gamma(2 \cdot 2-2+2)}\right]=\frac{1}{90}
\end{gathered}
$$




$$
\begin{gathered}
\mathcal{B}_{6}=(-1)^{4}\left[\frac{3}{\Gamma(2 \cdot 3+2)}+\frac{(-1) \cdot \mathcal{B}_{2}}{\Gamma(2 \cdot 3-2+2)}\right. \\
\left.+\frac{(-1)^{2} \cdot \mathcal{B}_{4}}{\Gamma(2 \cdot 3-2 \cdot 2+2)}\right] \\
=\frac{1}{945}
\end{gathered}
$$

$$
\begin{gathered}
\mathcal{B}_{8}=(-1)^{5}\left[\frac{4}{\Gamma(2 \cdot 4+2)}+\frac{(-1) \cdot \mathcal{B}_{2}}{\Gamma(2 \cdot 4-2+2)}+\frac{(-1)^{2} \cdot \mathcal{B}_{4}}{\Gamma(2 \cdot 4-2 \cdot 2+2)}\right. \\
\left.+\frac{(-1)^{3} \cdot \mathcal{B}_{6}}{\Gamma(2 \cdot 4-2 \cdot 3+2)}\right]=\frac{1}{9450}
\end{gathered}
$$

$$
\begin{aligned}
\mathcal{B}_{10}=(-1)^{6} & {\left[\frac{5}{\Gamma(2 \cdot 5+2)}+\frac{(-1) \cdot \mathcal{B}_{2}}{\Gamma(2 \cdot 5-2+2)}+\frac{(-1)^{2} \cdot \mathcal{B}_{4}}{\Gamma(2 \cdot 5-2 \cdot 2+2)}\right.} \\
& \left.+\frac{(-1)^{3} \cdot \mathcal{B}_{6}}{\Gamma(2 \cdot 5-2 \cdot 3+2)}+\frac{(-1)^{4} \cdot \mathcal{B}_{8}}{\Gamma(2 \cdot 5-2 \cdot 4+2)}\right]=\frac{1}{93555}
\end{aligned}
$$

Hence, the first five even zeta functions are as follows:-

$$
\begin{aligned}
\zeta(2) & =\frac{\pi^{2}}{6} \\
\zeta(4) & =\frac{\pi^{4}}{90} \\
\zeta(6) & =\frac{\pi^{6}}{945} \\
\zeta(8) & =\frac{\pi^{8}}{9450} \\
\zeta(10) & =\frac{\pi^{10}}{93555}
\end{aligned}
$$


R. Paul

\section{Conclusion}

In conclusion, one could alternatively use the deduced recursive relation to compute the zeta function for even integers. However, there is no closed form expression available for odd zeta functions and hence, these require much more intricate analysis unlike the even ones.

\section{References}

[1] George B Arfken and Hans J Weber. Mathematical methods for physicists 6th ed. Mathematical methods for physicists 6th ed. by George B. Arfken and Hans J. Weber. Published: Amsterdam; Boston: Elsevier, pages 376384, 2005.

[2] BILLY GENE CLAYBROOK. FOURIER SERIES AND THE FOURIER INTEGRAL. PhD thesis, KANSAS STATE UNIVERSITY, 1966. 